\title{
Self-Adjointness and Invariance of the Essential Spectrum for Dirac Operators Defined as Quadratic Forms
}

\author{
G. Nenciu \\ Institute of Atomic Physics, Bucharest, Romania
}

\begin{abstract}
Some general results about perturbations of not-semibounded selfadjoint operators by quadratic forms are obtained. These are applied to obtain the distinguished self-adjoint extension for Dirac operators with singular potentials (including potentials dominated by the Coulomb potential with $Z<137)$. The distinguished self-adjoint extension, is the unique selfadjoint extension, for which the wave functions in its domain possess finite mean kinetic energy. It is shown moreover that the essential spectrum of the distinguished extension is contained in the spectrum of the free Hamiltonian.
\end{abstract}

\section{Introduction}

In this paper we shall consider the problems of self-adjointness and of the invariance of the essential spectrum for the Dirac operator perturbed by a local potential. The formal Hamiltonian to be considered is

$$
-i \alpha \cdot \operatorname{grad}+m \beta+\boldsymbol{V}(x) .
$$

Reviews concerning the self-adjointness problem both in relativistic and nonrelativistic quantum mechanics appeared recently. See [2] where an extensive bibliography is also given, and [3] which considers also nonlocal perturbations. The status of the theory is almost satisfactory due to the results obtained in the last years. However, there is a point in which the relativistic theory is less satisfactory: the case of singular potentials when the minimal operator (1.1) is not essentially self-adjoint. In the nonrelativistic (Schrödinger) case the semiboundedness of the sesquilinear form defined by the minimal operator is sufficient to provide a distinguished self-adjoint operator in a canonical way, the Friedrichs extension, which is taken to represent the physical Hamiltonian irrespective of the fact that the minimal operator is essentially self-adjoint or not. In the relativistic case (due to the unboundedness of the free Dirac operator) a general method to 
construct distinguished self-adjoint extensions of the minimal operator (1.1) seems not to exist.

As the von Neumann theorem [5, Prop. 13.6] assures the existence of selfadjoint extensions of the formal operator (1.1) the problem is to provide a criterion to select one self-adjoint extension. The criterion must satisfy two requirements: first, to have a clear physical meaning; second, to assure the uniqueness i.e., in the cases of physical interest one have to prove that there exists only-one selfadjoint extension satisfying the criterion. In this paper we shall adopt the following criterion: the distinguished self-adjoint extension, $H$, satisfies

$$
\mathscr{D}(H) \subset \mathscr{D}\left(\left|H_{m}\right|^{1 / 2}\right)
$$

where $H_{m}$ is the free Dirac Hamiltonian (see Section 3). In the language of physics the condition (1.2) means that the force law has to determine the motion for states with finite kinetic energy. Then, the problem of self-adjointness is replaced by the problem of proving that there exists only one self-adjoint extension satisfying (1.2). There exists a method to construct self-adjoint extensions satisfying (1.2): the pseudo-Friedrich extension method [9, Th. 3.11], [5. Th. 5.2]. However, the pseudo-Friedrichs extension method is insufficient. Indeed, in the case when $V(x)=(v /|x|) 1$ the pseudo-Friedrichs extension method can be applied only for $|v|<2 / \pi$ while the exact results for this case [13] show that the whole $|v|<1$ region is to be covered. We shall show in Section 2 that in the perturbation theory of self-adjoint operators by quadratic forms, some results known in the semibounded case can be extended to the general one. This provides a general method, valid both in the Schrödinger and Dirac case, to define (and to prove the uniqueness of) the distinguished self-adjoint extension. Applying the theory to the case of potentials dominated by Coulomb like potentials we obtain the following result (see Section 5, Theorem 5.1 for the general result).

Let $\boldsymbol{V}(x)$ be the $4 \times 4$ symmetric matrix-valued function which represents the potential. If

$$
|x| \cdot|||\boldsymbol{V}(x) \|| \leqq v<1
$$

(here $\||\cdot| \mid$ means the usual $4 \times 4$ matrix norm) then there exists a unique self-adjoint extension $H$, of the formal operator (1.1), satisfying (1.2).

Others methods to construct self-adjoint extensions of the minimal Dirac operator (1.1) have been recently proposed by Schmincke [6] and Wüst [7]. Although, they were able to construct distinguished self-adjoint extensions for some classes of potentials containing $v /|x|,|v|<1$, their proofs are rather intricate and, more important their methods say nothing about the uniqueness of the distinguished self-adjoint extension. Regarding the generality it is easy to see that the class of potentials we shall consider in Section 5 is not contained in their class of potentials. On the other hand, although it seems not easy to decide if the classes of potentials considered in $[6,7]$ are fully contained in our class (actually the classes in $[6,7]$ are defined in a rather implicit manner) it is very likely that a more refined application of the general results contained in Section 2 of our paper will cover the results in $[6,7]$. Regarding the pseudo-Friedrichs method our method is strictly more general. More exactly the pseudo-Friedrichs method 
is based on the estimates on $\left\||V|^{1 / 2}\left(\left|H_{m}\right|+a\right)^{-1}|V|^{1 / 2}\right\|$ while our method rests on estimates on $\left\||V|^{1 / 2}\left(H_{m}+\lambda\right)^{-1}|V|^{1 / 2}\right\|$. From the physical point of view it is worthwhile to mention that, when definable, our distinguished self-adjoint extension coincide with that provided by the pseudo-Friedrichs method and by the method developed in [6] and [7]. Regarding the problem of the invariance of the essential spectrum, the usual method of proof that $\sigma_{\text {ess }}(H) \subset \sigma_{\text {ess }}\left(H_{m}\right)$ requires $V$ to be $H_{m}$ compact [9, IV.1.3 and IV.5.6]. This is not the case for singular potentials satisfying (1.2). We shall show that the $\left|H_{m}\right|^{3 / 2}$-compactness of $|V|^{1 / 2}$ is sufficient. This allows to prove that $\sigma_{\text {ess }}(H) \subset \sigma_{\text {ess }}\left(H_{m}\right)$ for all potentials satisfying (1.2). For another general result about invariance of the essential spectrum (requiring however $V$ to be $H_{m}$ vounded with bound smaller than one [9, IV.1.], which gives $v<1 / 2$ in (1.3)) see [3].

The paper is organized as follows. Section 2 contains the general theory of perturbation of nonsemibounded self-adjoint operators by symmetric sesquilinear forms. Section 3 contains the description of the free particle Dirac operator. Section 4 contains the description of the perturbing potential. Finally, Section 5 contains the applications of the general theory, developed in Section 2, to the Dirac operator.

\section{The General Theory}

Let $A$ be a self-adjoint operator in the separable Hilbert space $Y$. Let $A=U|A|=|A| U$ be the polar decomposition of $A$ [9, VI.2.7]. In contradistinction to [9] we shall define $U f=f$ for $f$ in the null subspace of $A$ so that $U$ is unitary and self-adjoint. Let for $\alpha>0, A_{\alpha}=A+\alpha U$. It is easy to see (using the functional claculus for selfadjoint operators) that

i) $A_{\alpha}$ is self-adjoint.

ii) $\mathscr{D}(A)=\mathscr{D}\left(A_{\alpha}\right) ; \mathscr{D}\left(|A|^{1 / 2}\right)=\mathscr{D}\left(\left|A_{\alpha}\right|^{1 / 2}\right)$.

iii) If $[-a, a]$ is in the resolvent set of $A$, then $[-\alpha-a, \alpha+a]$ is in the resolvent set of $A_{\alpha}$.

Let $X_{\alpha}$ be $\mathscr{D}\left(\left|A_{\alpha}\right|^{1 / 2}\right)$ with the Hilbert space structure given by

$$
(x, y)_{\alpha}=\left(\left|A_{\alpha}\right|^{1 / 2} x,\left|A_{\alpha}\right|^{1 / 2} y\right) ;\|x\|_{\alpha}^{2}=(x, x)_{\alpha}
$$

$X_{\alpha}$ are topologically equivalent as for $\alpha_{1}>\alpha_{2}>0$

$$
\|x\|_{\alpha_{2}} \leqq\|x\|_{\alpha_{1}} \leqq\left(\alpha_{1} / \alpha_{2}\right)^{1 / 2}\|x\|_{\alpha_{2}} .
$$

For an arbitrary self-adjoint operator $A$, its associated sesquilinear symmetric form, $h_{A}$, is defined by

$$
\begin{aligned}
\mathscr{Q}\left(h_{A}\right) & =\mathscr{D}\left(|A|^{1 / 2}\right), \\
h_{A}[x, y] & =\left(|A|^{1 / 2} x, U|A|^{1 / 2} y\right) ; x, y \in \mathscr{Q}\left(h_{A}\right) .
\end{aligned}
$$

It is easy to verify the following relation

$$
h_{A_{\alpha}}[x, y]=h_{A}[x, y]+\alpha(x, U y) .
$$

As $U \mathscr{D}\left(|A|^{1 / 2}\right)=\mathscr{D}\left(|A|^{1 / 2}\right)$ we can define the operator

$$
T=\left.U\right|_{\mathscr{D}\left(|A|^{1 / 2}\right)} .
$$


Because $U$ commutes with $\left|A_{\alpha}\right|^{1 / 2}, T$ is a unitary and self-adjoint operator on $X_{\alpha}$ and

$$
(x, T y)_{\alpha}=h_{A_{\alpha}}[x, y] .
$$

Let $v[x, y]$ a sesquilinear symmetric form in $Y$ with

$$
\mathscr{Z}(v) \supset \mathscr{D}\left(|A|^{1 / 2}\right) \text {. }
$$

If for some $\alpha, v$ is bounded on $X_{\alpha}$

$$
|v[x, y]| \leqq C_{\alpha}\|x\|_{\alpha} \cdot\|y\|_{\alpha}
$$

then it defines a bounded self-adjoint operator $V_{\alpha}$ by

$$
\left(V_{\alpha} x, y\right)_{\alpha}=v[x, y] \text {. }
$$

Definition 2.1. The symmetric sesquilinear form $v$ is a form perturbation of $A$ if it satisfies (2.8) and if there exists $\alpha>0$ such that (2.9) is satisfied and the operator $T+V_{\alpha}$ has bounded inverse in $X_{\alpha}$. If, for some $\alpha,\left\|V_{\alpha}\right\|<1$ then $v$ is a small form perturbation of $A$. If

$$
\lim _{\alpha \rightarrow \infty}\left\|V_{\alpha}\right\|=0
$$

then $v$ is tiny form perturbation of $A$.

Remarks. 1. If $v$ is a small form perturbation of $A$ then it is a perturbation of $A$.

2. If $v_{1}$ is a small form perturbation of $A$ and $v_{2}$ is a tiny form perturbation of $A$ then $v_{2}=v_{1}+v_{2}, \mathscr{2}\left(v^{2}\right)=\mathscr{Z}\left(v_{1}\right) \cap \mathscr{Q}\left(v_{2}\right)$ is a small form perturbation of $A$. This follows from the fact that $\left\|V_{\alpha}\right\|$ is a decreasing function of $\alpha$ due to (2.2) and (2.10).

3. The usual perturbation condition $[4,1,9]$

$$
|v[x, x]| \leqq a\left\||A|^{1 / 2} x\right\|^{2}+b\|x\|^{2} ; a<1, b<\infty ; x \in \mathscr{D}\left(|A|^{1 / 2}\right)
$$

implies that $v$ is a small form perturbation of $A$. Indeed (assuming that $a \neq 0$, otherwise there is nothing to prove) (2.12) can be written as

$$
|v[x, x]| \leqq a\left\|\left(|A|+a^{-1} b\right)^{1 / 2} x\right\|
$$

and it is sufficient to take $\alpha>a^{-1} b$.

Our first problem is to construct a self-adjoint operator $B$ whose associated sesquilinear symmetric form $h_{B}$ is the form sum (in a sense made precise below) of the forms $h_{A}$ and $v$. The following theorem provides such an operator.

Theorem 2.1. Let $A$ be a self-adjoint operator and $v$ a perturbation of $A$. Then, there exists a unique self-adjoint operator $B$ satisfying the condition

$$
\mathscr{D}(B) \subset \mathscr{D}\left(|A|^{1 / 2}\right)
$$

$$
(B x, y)=h_{A}[x, y]+v[x, y] ; \quad x \in \mathscr{D}(B), y \in \mathscr{D}\left(|A|^{1 / 2}\right) .
$$

Remarks. 4. For the case when $A \geqq 0$ and $v$ satisfies (2.12) the result is well known [4, App.], [9, VI.3]. Also, the result is essentially known for arbitrary $A$ 
if $v$ is a small form perturbation of $A$ [5, Th. 5.2]. Moreover, in these case

$$
\mathscr{D}\left(|A|^{1 / 2}\right)=\mathscr{D}\left(|B|^{1 / 2}\right) \text {. }
$$

The proof of the Theorem 2.1 rests on the following variant of the Lax-Milgram lemma [10, II. 1$]$.

Lemma 2.1. Let $\left\{X,(,)_{X},\|\cdot\|_{X}\right\},\left\{Y,(,)_{Y},\|\cdot\|_{Y}\right\}$ be two Hilbert spaces, $X \subset Y$, $\bar{X}=Y,\|\cdot\|_{Y} \leqq \delta\|\cdot\|_{X}, 0<\delta<\infty, C$ a bounded self-adjoint operator in $X$, with bounded inverse. Then, there exists a unique self-adjoint operator $\tilde{B}$ in $Y$ with $\mathscr{D}(\tilde{B}) \subset X$ and

$$
(\tilde{B} x, y)_{Y}=(C x, y)_{X} ; \quad x \in \mathscr{D}(\tilde{B}), y \in X .
$$

The proof of the lemma follows rather closely [10] (with the uniqueness argument borowed from [4]) and will be not given here.

Proof of the Theorem 2.1. We shall make the following realisation of the Lemma 2.1. $Y$ is the Hilbert space form Theorem 2.1. $X=X_{\alpha}$ given by (2.1), $C=T+V_{\alpha}$ given by (2.6) and (2.10). For the appropriate value of $\alpha$ the invertibility of $C$ is assumed by definition. Taking into account (2.5), the operator $B$ in the Theorem 2.1 is given by

$$
B=\tilde{B}-\alpha U \text {. }
$$

From the physical point of view the result contained in Theorem 2.1 has the inconvenience to be nonconstructive. The next result we shall prove, shows that the resolvent of $B$ is given by a formula which formally is the familiar Born series. In the remainder of this section we shall assume (although this is not essential) that $v$ is the associated sesquilinear form of a self-adjoint operator $V$ i.e. $v=h_{V}$. We shall use the following notation. If $G$ is a densely defined bounded operator in $Y$ we shall define by [G], its extension by continuity to the whole space. Let $V$ be $A$ form bounded [i.e. $h_{V}$ satisfies (2.12) for some $\left.a, b<\infty\right], \alpha>0, z \notin \sigma(A)$. Then the operators $|V|^{1 / 2}(A-z)^{-1},\left[(A-z)^{-1}|V|^{1 / 2}\right],\left[(|A|+\alpha)^{-1 / 2}|V|^{1 / 2}\right], M(z) \equiv$ $\left[|V|^{1 / 2}(A-z)^{-1}|V|^{1 / 2}\right]$ are bounded and

$$
\begin{aligned}
& {\left[(A-z)^{-1}|V|^{1 / 2}\right]=\left(|V|^{1 / 2}(A-\bar{z})^{-1}\right)^{*},} \\
& {\left[(|A|+\alpha)^{1 / 2}|V|^{1 / 2}\right]=\left(|V|^{1 / 2}(|A|+\alpha)^{-1 / 2}\right)^{*}} \\
& M(z)^{*}=M(\bar{z}) .
\end{aligned}
$$

Lemma 2.2. Let $A, V=S|V|$ be self-adjoint operators such that $V$ is $A$ form bounded and $h_{V}$ is a form perturbation of $A$. Let $B$ be the self-adjoint operator defined by Theorem 2.1. If for $z \notin \sigma(A), 1+S M(z)$ has a bounded inverse then $z \notin \sigma(B)$ and

$$
\begin{aligned}
(B-z)^{-1}= & (A-z)^{-1}-\left[(A-z)^{-1}|V|^{1 / 2}\right](1+S M(z))^{-1} \\
& \cdot S|V|^{1 / 2}(A-z)^{-1} \equiv R(z)
\end{aligned}
$$

Remarks. 5. If $\|M(z)\|<1$ then

$$
R(z)=(A-z)^{-1}-(A-z)^{-1} V(A-z)^{-1}+\ldots
$$

i.e. just the usual Born series. 
6. If $h_{V}$ is a small form perturbation of $A$ then for $z$ sufficiently far from $\sigma(A)$, $\|M(z)\|<1$ (it is sufficient to have $\left.|\operatorname{Im} z| \geqq(|\operatorname{Re} z|+\alpha) /(1-a)^{1 / 2}\right)$. For this case the result contained in Lemma 2.2 is known [5].

7. An example in which $V$ is not a small form perturbation of $A$ but Theorem 2.1 and Lemma 2.2 apply is provided by $A>0, V>0$ and $V$ is $A$ form bounded (take $z<0$ in Lemma 2.2).

Proof of Lemma 2.2. It is sufficient to prove that for every $x \in \mathscr{D}(B)$

$$
R(z)(B-z) x=x \text {. }
$$

Let us compute for arbitrary $y \in Y,(R(z)(B-z) x, y)$. Let us remark that $1+M(\bar{z}) S$ has a bounded inverse

$$
R^{*}(z)=R(\bar{z})
$$

and the operator $|A|^{1 / 2} R(z)$ is bounded which implies

$$
\operatorname{Ran} R(z) \subset \mathscr{D}\left(|A|^{1 / 2}\right) \text {. }
$$

Then using the condition $\left(\mathrm{L}_{2}\right)$

$$
\begin{aligned}
(R(z)(B-z) x, y)= & ((B-z) x, R(\bar{z}) y) \\
= & \left(|A|^{1 / 2} x, U|A|^{1 / 2} R(\bar{z}) y\right)+\left(|V|^{1 / 2} x, S|V|^{1 / 2} R(\bar{z}) y\right) \\
& -z(x, R(\bar{z}) y) .
\end{aligned}
$$

Now using the identity

$$
S M(z)(1+S M(z))^{-1}=1-(1+S M(z))^{-1}
$$

for $\beta>0$

$$
\begin{aligned}
\left(|V|^{1 / 2} x, S|V|^{1 / 2} R(\bar{z}) y\right) & =\left((|A|+\beta)^{1 / 2} x,(A-\bar{z})(|A|+\beta)^{-1 / 2}\left\{(A-\bar{z})^{-1}-R(\bar{z})\right\} y\right) \\
& =(x, y)-\left(|A|^{1 / 2} x, U|A|^{1 / 2} R(\bar{z}) y\right)+z(x, R(\bar{z}) y)
\end{aligned}
$$

From (2.23) and (2.25)

$$
(R(z)(B-z) x, y)=(x, y)
$$

which implies (2.20), $y$ being arbitrary in $Y$.

The following Lemma is the abstract form of an argument used in [8].

Lemma 2.3. Let $A, V$ as in Lemma 2.2. If for some $z_{0} \notin \sigma(A),(1+S M(z))$ has a bounded inverse and $\left[|V|^{1 / 2}\left(A-z_{0}\right)^{-1}(A-z)^{-1}|V|^{1 / 2}\right]$ is compact for $z \notin \sigma(A)$ then $(1+S M(z))^{-1}$ is a meromorphic operator valued function in $\mathbb{C} \backslash \sigma(A)$. In particular $(1+S M(z))$ has a bounded inverse everywhere in $\mathbb{C} \backslash \sigma(A)$ with the possible exception of a set having no accumulation points in $\mathbb{C} \backslash \sigma(A)$.

Remarks. 8. Lemma 2.3 is a generalization of the following well-known result about analytic families of compact operators.

Lemma 2.4 ([1,11,9]). Let $N(z)$ be an analytic compact operator valued function on a simply connected domain $D$. If for some $z_{0} \in D, 1+N\left(z_{0}\right)$ has a bounded inverse then $(1+N(z))^{-1}$ is a meromorphic operator valued function on $D$. 
Proof of Lemma 2.3. The proof is immediate

$$
\begin{aligned}
& 1+S M(z)=1+S M\left(z_{0}\right)+S\left(M(z)-M\left(z_{0}\right)\right) \\
& =\left(1+S M\left(z_{0}\right)\right)\left\{1+\left(z-z_{0}\right)\left(1+S M\left(z_{0}\right)\right)^{-1} S\left[|V|^{1 / 2}\left(A-z_{0}\right)^{-1}(A-z)^{-1}|V|^{1 / 2}\right]\right\} .
\end{aligned}
$$

Then taking

$$
N(z)=\left(z-z_{0}\right)\left(1+S M\left(z_{0}\right)\right)^{-1} S\left[|V|^{1 / 2}\left(A-z_{0}\right)^{-1}(A-z)^{-1}|V|^{1 / 2}\right] \lim _{z \rightarrow z_{0}}\|N(z)\|=0,
$$

$N(z)$ is compact and the application of Lemma 2.4 finishes the proof.

The following theorem gives examples of perturbations which are not necessarily small perturbations.

Theorem 2.2. Let $A, V$ be self-adjoint operators such that there exists $t \in \mathbb{R}$, $t \notin \sigma(A)$ and $V$ is $A$ form bounded. If $(1+S M(t))$ has a bounded inverse, then $h_{V}$ is a perturbation of $A-t$.

Proof. Without loss of generality we may assume that $t=0$ and $|A| \geqq 1$. Let us consider the following operator

$$
Q=U-\left[A^{-1}|V|^{1 / 2}\right](1+S M(0))^{-1} S|V|^{1 / 2} U .
$$

$Q$ is a bounded everywhere defined operator in $X_{0}\left(=\mathscr{D}\left(|A|^{1 / 2}\right)\right)$ with the norm $\|x\|_{0}=\left\||A|^{1 / 2} x\right\|$. Now

$$
\left(\left(T+V_{0}\right) x, Q y\right)_{0}=\left(|A|^{1 / 2} x, U|A|^{1 / 2} Q y\right)+\left(|V|^{1 / 2} x, S|V|^{1 / 2} Q y\right) .
$$

A calculation similar to that in the proof of the Lemma 2.2 gives

$$
\left(\left(T+V_{0}\right) x, Q y\right)_{0}=(x, y)_{0}
$$

i.e.

$$
Q^{*}\left(T+V_{0}\right)=1
$$

which implies that $T+V_{0}$ has a bounded inverse in $X_{0}$ and the proof of the theorem is finished.

Corollary 2.1. Let $A, V_{1}, V_{2}$, self-adjoint operators satisfying

i) $|A| \geqq m>0$.

ii) $V_{1}$ is $A$ form bounded and there exists $z_{0} \notin \sigma(A)$ such that

$$
\left(1+S_{1}\left[|V|^{1 / 2}\left(A-z_{0}\right)^{-1}|V|^{1 / 2}\right]\right)
$$

has a bounded inverse.

iii) $\left[\left|V_{1}\right|^{1 / 2}\left(A-z_{0}\right)^{-1}(A-z)^{-1}\left|V_{1}\right|^{1 / 2}\right]$ is compact for $z \notin \sigma(A)$.

iv) $V_{2}$ is a bounded operator and $V_{2}|A|^{-1 / 2}$ is compact. Then there exists a unique self-adjoint operator $B$ satisfying

$$
\begin{aligned}
& \mathscr{D}(B) \subset \mathscr{D}\left(|A|^{1 / 2}\right) \\
& (B x, y)=h_{A}[x, y]+h_{V_{1}}[x, y]+h_{V_{2}}[x, y] ; \quad x \in \mathscr{D}(B), y \in \mathscr{D}\left(|A|^{1 / 2}\right) .
\end{aligned}
$$


The operator $B$ has the property

$$
\sigma_{\mathrm{ess}}(B) \subset \sigma_{\mathrm{ess}}(A) \text {. }
$$

Proof. By Lemma $2.3\left(1+S_{1}\left[\left|V_{1}\right|^{1 / 2}(A-z)^{-1}\left|V_{1}\right|^{1 / 2}\right)^{-1}\right.$ is a meromorphic function on $\mathbb{C} \backslash \sigma(A)$. Then by Theorem 2.2, $h_{V_{1}}$ is a perturbation of $A-t$ for some $t \in(-m, m)$. Let $\tilde{\tilde{B}}=A-t+V_{1}$ in the sense of Theorem $2.1, \tilde{B}=\tilde{\tilde{B}}+t$ and $B=\tilde{B}+V_{2}$. Obviously

$$
(\tilde{\tilde{B}}-z)^{-1}=(\tilde{B}-(z-t))^{-1}
$$

and from Lemma 2.2 and 2.3

$$
\sigma_{\text {ess }}(\tilde{\tilde{B}}) \subset \sigma_{\text {ess }}(A-t) \text {. }
$$

For sufficiently large $\operatorname{Im} z$

$$
(B-z)^{-1}=(\tilde{\tilde{B}}-(z+t))^{-1}\left\{1+V_{2}(\tilde{\tilde{B}}-(z+t))^{-1}\right\}^{-1} .
$$

On the other hand using (2.18) for $(\tilde{\tilde{B}}-z)^{-1}$ and iv), it follows that $V_{2}(\tilde{\tilde{B}}-z)^{-1}$ is compact and (2.33) follows from Lemma 2.3.

Remarks. 9. In the theory developed in this section we have used for the sake of simplicity the factorisation

$$
V=S|V|^{1 / 2}|V|^{1 / 2} \text {. }
$$

The whole theory can be developed with a general factorisation

$$
V=V_{1} V_{2}
$$

with $V_{1}, V_{2}-|A|^{1 / 2}$ bounded.

\section{The Free-particle Dirac Hamiltonian}

Let $\left(L^{2}\right)^{4}$ be the Hilbert space of the $\mathbb{C}^{4}$-valued functions, $\Psi(x) \equiv\left(\psi_{i}(x)\right)_{i=1}^{4}$, of the space variable $x=\left(x_{1}, x_{2}, x_{3}\right)$ with the usual scalar product

$$
(\Psi, \Phi)=\sum_{i=1}^{4} \int \psi_{i}(x) \overline{\varphi_{i}(x)} d x .
$$

The specification of the integration domain will be dropped whenever the integrals are extended over the whole $\mathbb{R}^{3}$ space.

The Hamiltonian $H_{m}$ of a free Dirac particle of mass $m$ is given by the selfadjoint extension in $\left(L^{2}\right)^{4}$ of the following differential operator [9, V.5.4]

$$
-i \sum_{k=1}^{3} \alpha_{k} \partial / \partial x_{k}+m \beta ; \quad \alpha_{k}=\left(\begin{array}{cc}
0 & \sigma_{k} \\
\sigma_{k} & 0
\end{array}\right), \quad \beta=\left(\begin{array}{cc}
1_{2} & 0 \\
0 & -1_{2}
\end{array}\right)
$$

where $\sigma_{1}, \sigma_{2}, \sigma_{3}$ are the Pauli $2 \times 2$ matrices and $1_{2}$ is the $2 \times 2$ unit matrix. $H_{m}$ has only absolute continuous spectrum

$$
\sigma\left(H_{m}\right)=(-\infty,-m] \cup[m, \infty) .
$$


The integral kernel of the resolvent $\left(H_{m}-E\right)^{-1}$ is given by

$$
G_{0}(x, y ; E)=\left(-i \sum_{k=1}^{3} \alpha_{k} \partial / \partial x_{k}+m \beta+E\right)(4 \pi)^{-1} \exp (i \lambda|x-y|) /|x-y|
$$

where

$$
\lambda^{2}=E^{2}-m^{2} ; \quad \operatorname{Im} \lambda \geqq 0 .
$$

\section{The Potential}

Let $V(x)$ a $4 \times 4$ symmetric matrix, whose elements $V_{i j}(x)$ are (complex valued) measurable a.e. finite functions on $\mathbb{R}^{3}$. Exactly as in the scalar case one can show that

$$
\begin{aligned}
& (V \Psi)_{i}(x)=\sum_{j} V_{i j}(x) \psi_{j}(x), \\
& \mathscr{D}(V)=\left\{\left.\Psi \in\left(L^{2}\right)^{4}\left|\sum_{i} \int\right|(V \Psi)_{i}(x)\right|^{2} d x<\infty\right\}
\end{aligned}
$$

is a self-adjoint operator in $\left(L^{2}\right)^{4}$. Let $x_{0} \in \mathbb{R}^{3}$ such that $\left|V_{i j}\left(x_{0}\right)\right|<\infty$. Then $V\left(x_{0}\right)$ is a symmetric matrix which admits a polar decomposition

$$
\boldsymbol{V}\left(x_{0}\right)=\boldsymbol{S}\left(x_{0}\right)|\boldsymbol{V}|\left(x_{0}\right)
$$

where $|\boldsymbol{V}|\left(x_{0}\right)$ is a positive definite matrix, and $\boldsymbol{S}\left(x_{0}\right)$ is a symmetric unitary matrix.

Let

$$
V=S|V|
$$

be the polar decomposition of $V$. Then

$$
(|V| \Psi)_{i}(x)=\sum_{j}|V|_{i j}(x) \Psi_{j}(x)
$$

Let $\left\|V\left(x_{0}\right)\right\| \mid$ be the usual $4 \times 4$ matrix norm (the greatest eigenvalue of $|\boldsymbol{V}|\left(x_{0}\right)$. Then

$$
\|V\|=\underset{x \in \mathbb{R}^{3}}{\operatorname{ess}-\sup }\|\boldsymbol{V}(x)\| \| .
$$

\section{Dirac Operators Defined as Quadratic Forms}

In this section we shall apply the general theory developed in Section 2 to the case when $Y=\left(L^{2}\right)^{4}, A=H_{m}$ given by (3.3), (3.4) and $V$ is given by (4.1), (4.2).

Let $w(t)$ be a decreasing function on $[0, \infty)$

$0 \leqq w(t) \leqq 1 ; \quad \lim _{t \rightarrow \infty} w(t)=0$.

Lemma 5.1. Let us suppose that

$$
\boldsymbol{V}(x)=w(|x|) \boldsymbol{W}(x)
$$

where $W$ is $H_{m}$ form bounded. Then $\left[|V|^{1 / 2}\left(H_{m}-E_{0}\right)^{-1}\left(H_{m}-E\right)^{-1}|V|^{1 / 2}\right], E$, $E_{0} \notin \sigma\left(H_{m}\right)$ is compact. 
Proof.

$$
\begin{aligned}
& {\left[|V|^{1 / 2}\left(H_{m}-E_{0}\right)^{-1}\left(H_{m}-E\right)^{-1}|V|^{1 / 2}\right]} \\
& \quad=\left(|V|^{1 / 2}\left|H_{m}\right|^{-1 / 2}\right)\left(\left|H_{m}\right|^{2}\left(H_{m}-E_{0}\right)^{-1}\left(H_{m}-E\right)^{-1}\right)\left[\left|H_{m}\right|^{-3 / 2}|V|^{1 / 2}\right]
\end{aligned}
$$

so that it is sufficient to prove that $\left[\left|H_{m}\right|^{-3 / 2}|V|^{1 / 2}\right]$ is compact. Now for $\alpha>0$ [14]

$$
\left|H_{m}\right|^{-\alpha}=\left(-\Delta+m^{2}\right)^{-\alpha / 2}
$$

i.e. the integral kernel of $\left|H_{m}\right|^{-\alpha}$ is essentially the Bessel potential $[12, \mathrm{~V}$.$] . Because$ of (5.2) it is easy to see that

$$
\lim _{n \rightarrow \infty}\left\|\left[\left|H_{m}\right|^{-3 / 2}\left(1-\chi_{n}(\cdot)\right)|V|^{1 / 2}\right]\right\|=0
$$

where

$$
\chi_{n}(x)= \begin{cases}1 ; & |x| \leqq n \\ 0 ; & |x|>n\end{cases}
$$

so that it is sufficient to prove that $\left[\left|H_{m}\right|^{-3 / 2} \chi_{n}(\cdot)|V|^{1 / 2}\right]$ is compact for all positive integers $n$ (the norm limit preserves compactness). Using the elementary properties of the Bessel potential $[12, \mathrm{~V} .3]$ one can see that there exists $c>0$ such that $\left[\exp (c|\cdot|)\left|H_{m}\right|^{-1 / 2} \chi_{n}(\cdot)|V|^{1 / 2}\right]$ is a bounded operator so that it is sufficient to prove that $\left|H_{m}\right|^{-1} \exp (-c|\cdot|)$ is compact. Now the compactness of $\left|H_{m}\right|^{-1}$ $\cdot \exp (-c|\cdot|)$ follows from elementary properties of double scale of weighted $L^{2}$ spaces [15, property (4)].

Let

$$
V_{c, v}(x)=(v /|x|) 1 \quad v \geqq 0
$$

the Coulomb potential. The inequality [9, p. 307]

$$
\left(\Phi,|\cdot|^{-1} \Phi\right) \leqq(\pi / 2)\left(\Phi,\left|H_{m}\right| \Phi\right), \quad \Phi \in \mathscr{D}\left(H_{m}\right)
$$

shows that $V_{c, v}$ is form bounded for all $0<v<\infty$ and that for $0<v<2 / \pi, h_{V_{c, v}}$ is a small perturbation of $H_{m}$.

Let us remark that

$$
|x|^{-1}=\left(|x|^{-1}+1\right)(1+|x|)^{-1}
$$

which shows that $V_{c, v}$ satisfies the conditions of Lemma 5.1. In the following we shall denote by $k_{s}, r^{\alpha}$ the operators of multiplication with $k_{s}(x)$ and $\left(x_{1}^{2}+x_{2}^{2}+x_{3}^{2}\right)^{\alpha / 2}$ respectively. The technical result we need about $V_{c, v}$ is contained in the following.

Lemma 5.2. Let $s>0$ and

$$
k_{s}(x)=\left\{\begin{array}{lll}
\left(1-(m+s)|x|+\left(m^{2}+s^{2}\right)|x|^{2},\right. & \text { for } & |x| \leqq(m+s) /\left(m^{2}+s^{2}\right) \\
1 & \text { for } & |x|>(m+s) /\left(m^{2}+1^{2}\right) .
\end{array}\right.
$$

Then

$$
\left\|\left[k_{s} r^{-1 / 2}\left(H_{m} \pm i s\right)^{-1} r^{-1 / 2} k_{s}\right]\right\| \leqq 1 .
$$

Remarks. 9. The result contained in Lemma 5.2 is a generalization of Lemma 2 in [7]. 
10. We conjecture the more aesthetic inequality

$$
\left\|\left[r^{-1 / 2}\left(H_{m}+i s\right)^{-1} r^{-1 / 2}\right]\right\| \leqq 1 .
$$

Proof of Lemma 5.2. Let

$$
\mathscr{D}_{0}=\left\{\Phi \mid \varphi_{i} \in \mathrm{C}_{0}^{\infty}\left(\mathbb{R}^{3} \backslash\{0\} .\right.\right.
$$

The following operators are symmetric on $\mathscr{D}_{0}$

$$
P_{k}=-i \partial / \partial x_{k}
$$

$x_{k}=$ the multiplication with $x_{k}$

$L_{i}=\sum_{j, k=1}^{3} \varepsilon_{i j k} x_{j} P_{k}, i=1,2,3$ where $\varepsilon_{i j k}=1$ if $i j k$ is a circular permutation of $\{1,2,3\}$ and zero otherwise

$$
\begin{aligned}
\Sigma_{l} & =i \sum_{j, k=1}^{3} \varepsilon_{l j k} \alpha_{j} \alpha_{k}, \quad l=1,2,3 \\
P_{r} & =r^{-1}\left(\sum_{k=1}^{3} x_{k} P_{k}-i 1\right) \\
L_{\Sigma} & =\sum_{k=1}^{3} \sum_{k} L_{k} \quad L^{2}=\sum_{k=1}^{3} L_{k}^{2} \\
\alpha_{r} & =r^{-1} \sum_{k=1}^{3} \alpha_{k} x_{k} \\
\alpha P & =\sum_{k=1}^{3} \alpha_{k} P_{k} .
\end{aligned}
$$

The following identities and inequalities hold on $\mathscr{D}_{0}[6]$.

$$
\begin{aligned}
& \alpha_{r} \cdot \alpha_{r}=1, \\
& (\alpha P) r-r(\alpha P)=-i \alpha_{r}, \\
& (\alpha P) r(\alpha P)=P_{r} r P_{r}+r^{-1}\left(L^{2}+L_{\Sigma}\right)+r^{-1}, \\
& (\alpha P) r \beta+\beta r(\alpha P)=i \beta \alpha_{r}, \\
& r^{-1}\left(L^{2}+L_{\Sigma}\right) \geqq 0 .
\end{aligned}
$$

Let $\Phi \in \mathscr{D}_{0}$. Using (5.15-19) we have

$$
\begin{aligned}
\left\|r^{1 / 2}\left(H_{m} \pm i s\right) \Phi\right\|^{2}= & \left(\Phi,\left(H_{m} \mp i s\right) r\left(H_{m} \pm i s\right) \Phi\right) \\
= & \left(\Phi, P_{r} r P_{r} \Phi\right)+\left(\Phi, r^{-1}\left(L^{2}+L_{\Sigma}\right) \Phi\right)+\left(\Phi, r^{-1} \Phi\right) \\
& +\left(\Phi, i m \beta \alpha_{r} \Phi\right)+\left(\Phi, s \alpha_{r} \Phi\right)+\left(s^{2}+m^{2}\right)(\Phi, r \Phi) \\
\geqq & \left(\Phi,\left\{r^{-1}-(s+m)+\left(s^{2}+m^{2}\right) r\right\} \Phi\right) \geqq\left\|k_{1} r^{-1 / 2} \Phi\right\|^{2} .
\end{aligned}
$$

Obviously $k_{s} r^{-1 / 2} \mathscr{D}_{0}=\mathscr{D}_{0}, r^{1 / 2} k_{s}^{-1} \mathscr{D}_{0}=\mathscr{D}_{0}$. Let $\Psi \in \mathscr{D}_{0}$. Then from (5.20)

$$
\left\|k_{s}^{-1} r^{1 / 2}\left(H_{m} \pm i s\right) r^{1 / 2} k_{s}^{-1} \Psi\right\| \geqq\left\|r^{1 / 2}\left(H_{m} \pm i s\right) r^{1 / 2} k_{s}^{-1} \Psi\right\| \geqq\|\Psi\|
$$

which proves the lemma. 
Definition 5.1. We shall say that $V$ is dominated by $V_{c, v}$ if $\|\boldsymbol{V}(x)\||\leqq v /| x \mid, \quad x \in \mathbb{R}^{3} \backslash\{0\}$.

We shall say that $V$ is nonsingular if $h_{|V|}$ is a tiny perturbation of $H_{m}$.

Remarks. 11. If $V_{i j} \in L^{p}\left(\mathbb{R}^{3}\right), p>3 ; \quad i, j=1, \ldots, 4$ then $V$ is nonsingular. Actually in this case, using (3.4) and Hölder and Young inequalities, one can prove that [14]

$$
\lim _{s \rightarrow \infty}\left\|\left[\left(H_{m}+i s\right)^{-1}|V|\right]\right\|=0
$$

which implies $[5$, p. 31$]$

$$
\lim _{s \rightarrow \infty}\left\|\left[\left(\left|H_{m}\right|+s\right)^{-1 / 2}|Y|^{1 / 2}\right]\right\|=0 .
$$

We are now ready to state the main result of this section.

Theorem 5.1. Let $m>0, w(t)$ satisfying (5.1) and $H_{m}, V$ defined by (3.3-4) and (4.1-2) respectively.

If either

a) $\boldsymbol{V}(x)=w(|x|) \boldsymbol{W}(x)$

where $W$ is a small form perturbation of $\mathrm{H}_{m}$. Or

b) $V(x)=V_{1}(x)+V_{2}(x)$

where $V_{1}$ is dominated by $V_{c, v}$ for some $v<1$ and

$$
\boldsymbol{V}_{2}(x)=w(|x|) \boldsymbol{W}_{2}(x)
$$

where $W_{2}$ is nonsingular.

Then

i) There exists a unique self-adjoint operator $H$ such that

$\mathscr{D}(H) \subset \mathscr{D}\left(\left|H_{m}\right|^{1 / 2}\right)$,

$(H \Phi, \Psi)=h_{H_{m}}[\Phi, \Psi]+h_{V}[\Phi, \Psi], \quad \Phi \in \mathscr{D}(H), \Psi \in \mathscr{D}\left(\left|H_{m}\right|^{1 / 2}\right)$,

ii)

$$
\sigma_{\text {ess }}(H) \subset \sigma_{\text {ess }}\left(H_{m}\right) \text {. }
$$

Proof. The first case is a direct consequence of Corrolary 2.1, Lemma 5.1 and Remark 6. The proof of the second case is somewhat more involved. Let us write

$$
\boldsymbol{V}_{1}(x)=k_{t}^{2}(x) \boldsymbol{V}_{1}(x)+\left(1-k_{t}^{2}(x)\right) \boldsymbol{V}_{1}(x)
$$

where $k_{t}(x)$ is defined by (5.10) and $t \in \mathbb{R}^{+}$is to be chosen later. Let us remark that

$$
\begin{aligned}
& \left.\left\|k_{t}(x)\left|V_{1}\right|^{1 / 2}(x)\right\|\left|\leqq v^{1 / 2} k_{t}(x)\right| x\right|^{-1 / 2}, \\
& \left\|\left(1-k_{t}^{2}(x)\right) V_{1}(x)\right\| \| v(m+t), \\
& \left(1-k_{t}^{2}(x)\right) V_{1}(x) \equiv 0 \text { for }|x|>(m+t) /\left(m^{2}+t^{2}\right) .
\end{aligned}
$$

Let

$$
\boldsymbol{V}_{t}(x)=k_{t}^{2}(x) \boldsymbol{V}_{1}(x)+V_{2}(x) .
$$


In view of Corrolary 2.1, Lemma 5.1, and Remark 9 is sufficient to prove that there exists $t \in[0, \infty)$ such that

$$
\boldsymbol{V}_{t}(x)=\boldsymbol{T}_{1}(x) \boldsymbol{T}_{2}(x) ;\left\|\left[T_{i}\left|H_{m}\right|^{-1 / 2}\right]\right\|<\infty ; \quad i=1,2
$$

and

$$
\left\|\left[T_{1}\left(H_{m}+i t\right)^{-1} T_{2}\right]\right\|<1 .
$$

A factorisation satisfying (5.36) is given by

$$
\boldsymbol{V}_{t}(x)=\left(k_{t}(x)\left\|\left|\boldsymbol{V}_{1}(x) \|^{1 / 2}+\right| \boldsymbol{V}_{2} \mid(x)^{1 / 2}\right)\left(\left(k_{t}(x) \boldsymbol{V}_{1}(x) /\left\|\boldsymbol{V}_{1}(x)\right\| \|^{1 / 2}\right)+\boldsymbol{Y}(x)\right)\right.
$$

where

$$
\boldsymbol{Y}(x)=\left(k_{t}(x)\|\| \boldsymbol{V}_{1}(x)\|\|^{1 / 2}+\left|\boldsymbol{V}_{2}\right|^{1 / 2}(x)\right)^{-1}\left(\boldsymbol{V}_{2}(x)-\left|\boldsymbol{V}_{2}\right|(x)^{1 / 2} k_{t}(x) \boldsymbol{V}_{1}(x) /\left\|\boldsymbol{V}_{1}(x)\right\| \|^{1 / 2}\right) .
$$

One can easily see that

$$
\lim _{s \rightarrow \infty}\left\|\left[\left(\left|H_{m}\right|+s\right)^{-1 / 2} Y\right]\right\|=0
$$

which together with Lemma (5.2) assures the existence of $t \in[0, \infty)$ such that (5.37) is true and the theorem is proved.

Acknowledgements. It is a pleasure for the author to thank Professor C. Foias for his interest in this work, and Drs. N. Angelescu, M. Bundaru and I. Raszillier for many useful discussions. I am grateful to the referee for poiting out an error in the initial form of the proof of Theorem 5.1.

\section{References}

1. Simon, B.: Quantum mechanics for Hamiltonians defined as quadratic forms. Princeton University Press 1971

2. Kalf,H., Schmincke, U.-W., Walter, J., Wüst, R.: On the spectral theory of Schrödinger and Dirac operators with singular potentials. Symposion on Spectral Theory and Differential Equations. Dundee 1974

3. Jörgens, K.: Perturbations of Dirac operator. Lecture Notes in Mathematics, Vol. 280. BerlinHeidelberg-New York: Springer 1973

4. Nelson, E.: J. Math. Phys. 5, 1190-1197 (1964)

5. Faris, W.G.: Self-adjoint operators. Lecture Notes in Mathematics, Vol. 433. Berlin-HeidelbergNew York: Springer 1975

6. Schmincke, U.-W.: Math. Z. 129, 335-349 (1972)

7. Wüst, R.: Distinguished self-adjoint extensions of Dirac operators by means of cut-off potentials. Aachen Preprint 1974

8. Nenciu, G.: Commun. math. Phys. 42, 221-229 (1975)

9. Kato, T.: Perturbation theory for linear operators. Berlin-Heidelberg-New York: Springer 1966

10. Lions, J. L.: Equations differentielles opérationnelles et problèmes aux limites. Berlin-GöttingenHeidelberg: Springer 1961

11. Hunziker, W.: Helv. Phys. Acta 39, $451-462$ (1966)

12. Stein, E. M.: Singular integrals and differentiability properties of functions. Princeton: Princeton University Press 1970

13. Narnhofer, H.: Acta Phys. Austr. 40, 306-322 (1974)

14. Prosser, R. T.: J. Math. Phys. 4, 1048-1054 (1963)

15. Prosser, R. T.: A double scale of weighted $L^{2}$ spaces. Bull. A.M.S. 81, 615-618 (1975)

Communicated by W. Hunziker

Received April 15, 1975; in revised form November 10, 1975 
